615

\section{CHANGES IN MYOCARDIAL VELOCITIES AND HAEMODYNAMICS AFTER SURGICAL LIGATION OF THE PATENT DUCTUS ARTERIOSUS IN PRETERM INFANTS}

\section{N. Patel}

Neonatal Intensive Care Unit, Royal Hospital for Sick Children, Glasgow, UK

Background and aims: The circulatory effects of surgical ligation of a haemodynamically significant ductus arteriosus (DA) in a preterm infant are poorly understood. This study aimed to measure changes in myocardial velocities, as an assessment of ventricular function, following ligation of the DA, using the new technique of Pulsed wave tissue Doppler imaging (PWTDI).

Methods: Echocardiograms were performed within 24 hours before and after DA ligation. Myocardial velocities were measured in the basal left and right ventricles and inter-ventricular septum (RV, LV \& IVS). Duct size and flow, left atrium:aortic valve ratio (LA:Ao), and left ventricular output (LVO) were also measured.

Results: Data was collected in five infants, median weight 1.4 (range 0.7-2.1) kg, gestation 26.0 (24.430.3) weeks, age 25 (22-50) days. Infants were ventilated, none received inotropes. PDA diameter was $0.30(0.20-0.33) \mathrm{cm}$, ductal flow was left to right. Following DA ligation, LVO was reduced (mean [SD], 335[118] vs. 258 [63] $\mathrm{mL} / \mathrm{kg} / \mathrm{min}$ ). LA:Ao was reduced but remained above normal limits (2.2[0.3] vs.1.9[0.4]). There was a trend of reduced mean LV myocardial velocities after DA ligation, however there was considerable variation between infants (Figure 1).

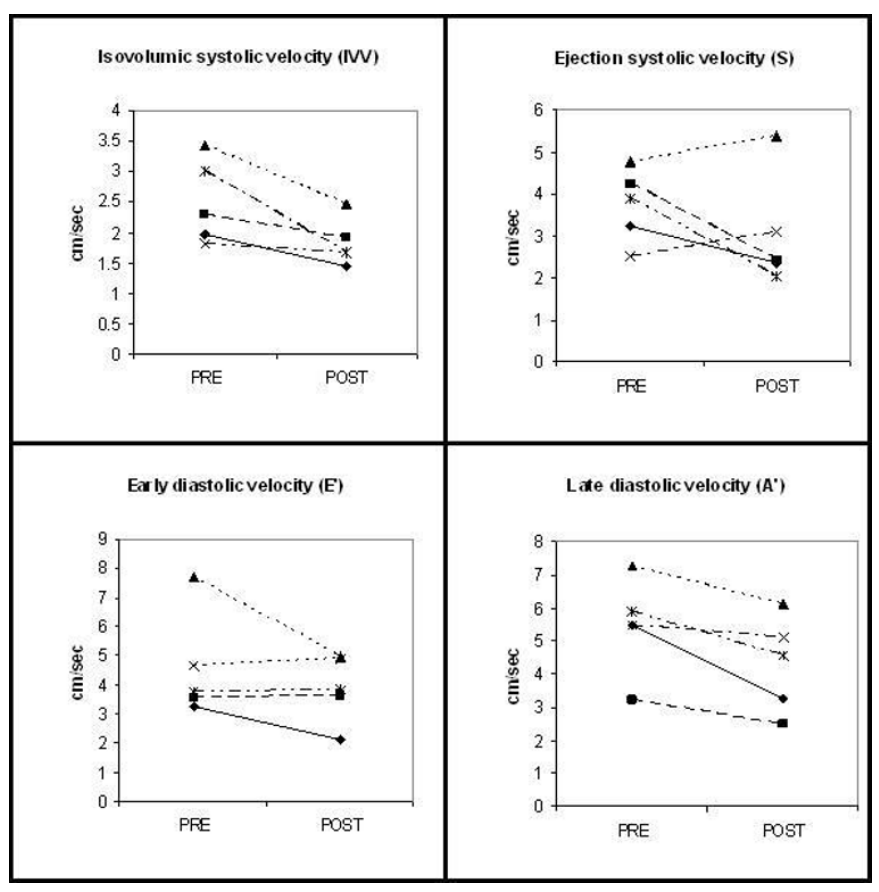

[Figure 1: Left ventricular myocardial velocities, ]

Conclusions: DA ligation acutely reduces LVO, altering loading conditions in the left heart. Reduced mean myocardial velocities in the LV may represent a reduction in both systolic and diastolic function after DA ligation. Individual variation in myocardial response may explain why some, but not all, infants experience cardio-respiratory instability after DA ligation.

\section{6}

\section{TAKO-TSUBO CARDIOMYOPATHY IN TEENAGERS}

G. Senatorova, I. Stenkova, N. Makieieva

Pediatrics and Neonatology, Kharkiv National Medical University, Kharkiv, Ukraine

Background: Tako-Tsubo cardiomyopathy (also called transient left ventricular apical ballooning or "broken heart" syndrome) is transient myocardial pathology, which mimics acute coronary syndrome, acute myocardial infarction with the ST-segment elevation on electrocardiogram without the coronary arteries damage. In Japanese, "tako-tsubo" means "fishing pot for trapping octopus," because the left ventricle of the patient with this condition resembles it. As there are only few descriptions of current syndrome we decided to report of our study.

Methods and results: 3 teenagers complaining of acute chest pain, palpitation, dyspnea, suddenly arising up after acute emotional and physiological 
stress (serious quarrel with classmates, physical activities) have been observed. The patients had no coronary risk factors. Their average blood pressure was $150 / 90 \mathrm{mmHg}$ and heart rate was 120 beats/ min. ST-segment elevations and T-wave inversions were registered on electrocardiograms of teenagers. Dysfunction and dyskinesia of the left ventricle apex and interventricular septum was detected on echocardiography. The levels of creatine kinase$\mathrm{MB}$ and troponin I did not increase. Occlusions and anomalies of vessels were not demonstrated by aortocoronarography. Tako-Tsubo cardiomyopathy was diagnosed in all cases. Symptomatic medical treatment had been administered and the function of heart was normalized within 3-4 weeks.

Conclusions: Tako-Tsubo cardiomyopathy may occur not only in adults, but in teenagers too. Therefore increased awareness of Tako-Tsubo cardiomyopathy will lead to its more frequent diagnosing, which will result in adequate medical management.

\section{7}

\section{OUTCOME OF MURMURS BEYOND INFANCY}

D.K. Gandhi ${ }^{1}$, P. Gali ${ }^{1}$, S. Sivakumar ${ }^{2}$

${ }^{1}$ Paediatrics, ${ }^{2}$ Paediatrics and Neonates, Sandwell and West Birmingham Hospitals NHS Trust, Birmingham, UK

Aim: Aim is to find out outcome of children above 1 referred with murmurs.

Methods: This is a retrospective audit of patients above 1 referred between April 2007 and October 2009 to consultant paediatrician with expertise in cardiology at a paediatric cardiac clinic in a district general hospital.

Results: 190 of $700+$ seen in cardiac clinic were children above 1 year of age referred for murmur \pm associated problems. 55 were from General Practitioners (29\%) and 135 from Paediatric colleagues (71\%). Murmur resolved in 16\% (31/190) proportionally from each group. Of remaining 159 children with persistent murmur, clinical examination and echocardiography confirmed innocent murmur in $140(88 \%)$ and they were discharged. 19 children (12\% of each group) were diagnosed to have congenital heart disease. Cardiac diagnoses were Ventricular Septal Defect (8), AtrioVentricular Septal Defect (1), Patent Ductus Arteriosus (2), Atrial Septal Defect (3), Aortic Stenosis (3) and Branch Pulmonary Stenosis( 2). 6 of these $(31 \%)$ with significant lesions were referred to tertiary cardiac centre for further assessment/intervention.

Conclusion: Childhood murmurs are sometimes transient and vast majority of persistent murmurs are innocent. Asymptomatic murmurs after infancy could be reviewed by paediatricians and persistent or symptomatic ones referred to paediatric cardiac clinic. Facility to perform an echocardiography at district general hospital will help in confirming innocent murmurs and more importantly reassuring anxious parents. It also has a role in detecting few, yet significant, congenital heart conditions that could then be referred on to tertiary centre.

\section{8}

\section{SIGNIFICANCE OF MURMURS IN INFANCY}

\author{
V. Gali ${ }^{1}$, D.K. Gandhi ${ }^{2}$, S. Sivakumar ${ }^{1}$,
} R. Sathanantharajah ${ }^{1}$

${ }^{1}$ Neonatal Unit, Birmingham City Hospital, Sandwell and West Birmingham Hospitals NHS

Trust, Birmingham, ${ }^{2}$ Department of Paediatrics,

Sandwell Hospital, Sandwell and West Birmingham Hospitals NHS Trust, West Bromwich, UK

Aim: To find out the significance of murmurs heard during infancy.

Methods: Retrospective audit of all infants with newly heard murmurs referred from April 2007 to October 2009 to cardiac clinics in district general hospital run by a Paediatrician with special expertise in cardiology.

Results: Over 30 month period, 246 infants were newly referred for murmurs. No murmurs were heard in clinic in $36 \%(88 / 246)$. Of 158 persistent murmurs, 54 were discharged following normal echocardiogram. Abnormalities excluding Patent Foramen Ovale (PFO) on echocardiogram were found in 91 (58\%). These were Ventricular Septal Defect (VSD) 34 (37\%), Pulmonary Branch Stenosis 24 (26\%), Patent ductus arteriosus (PDA) 7, Atrial Septal Defect 7, PFO \& PDA 6, Aortic Stenosis 5, VSD \& PDA 1 and Tetralogy of Fallot 2, Pulmonary Stenosis 1, Transposition of great arteries (TGA) 1, Congenitally Corrected TGA 1, Double Outlet Right ventricle 1, Complete Atrio-Ventricular Septal defect 1.24 of the 91 infants $(26 \%)$ were referred to the nearest tertiary cardiac centre for further management. Abnormalities in ECG were seen in $20 / 24$ infants. 80 infants with less significant cardiac defects did not require any intervention and were followed up locally. 